\title{
Carcinomatose meníngea nos tumores sólidos
}

\author{
Meningeal carcinomatosis in solid tumours
}

\author{
Volney Soares Lima' , Amândio Soares Fernandes Jr. ${ }^{2}$, Roberto Porto Fonseca² e Stella Sala Soares Lima ${ }^{3}$
}

\section{Resumo}

$\mathrm{O}$ acometimento metastático das leptomeninges e do líquor por células neoplásicas pode ser definido como carcinomatose meníngea ou meningite carcinomatosa. Nos últimos 20 anos tem sido observado um aumento significativo da sua incidência, atribuído principalmente ao melhor controle da doença sistêmica com terapias mais efetivas. A neoplasia mais freqüentemente associada à carcinomatose meníngea é o câncer de mama, seguido pelo câncer de pulmão e melanoma. A disseminação de células malignas pelo espaço sub-aracnóideo produz uma série de sinais e sintomas neurológicos pleomórficos, caracterizados principalmente pelo acometimento de todos os níveis do neuroeixo. A suspeição clínica e o diagnóstico precoce são fundamentais para uma melhor preservação das funções neurológicas e da qualidade de vida. A presença de metástases leptomeníngeas de tumores sólidos é complicação associada a prognóstico reservado, com sobrevida mediana de 2 a 4 meses. O tratamento é essencialmente paliativo, incluindo radioterapia, quimioterapia intratecal e quimioterapia sistêmica. É crucial uma seleção adequada dos pacientes com maiores chances de se beneficiarem de um tratamento mais agressivo. Este artigo consiste numa revisão da literatura sobre este tema, feita através de pesquisa em banco de dados do MEDLINE de 1970 a 2002.

Palavras-chave: tumores sólidos; leptomeninges; meningite carcinomatosa.

\section{Abstract}

Leptomeningeal and CSF neoplastic cell metastatic involvement may be defined as meningeal carcinoma or carcinomatous meningitis. A significantly increased incidence has been observed in the last 20 years, generally attributed to improved control of the systemic disease with more effective therapy. Breast cancer is the most common malignancy associated with menigeal carcinomatosis, followed by lung cancer and melanoma. Dissemination of malignant cells in the subaracnoid space produces pleomorphic neurologic signs and symptoms, usually characterized by involvement of all neurological levels. Clinical suspicion and early diagnosis are essential to preserve neurological functions and quality of life. Leptomeningeal solid tumor involvement is associated with an unfavorable progression, usually a mean 2 to 4 month survival period. Treatment is essentially palliative, including radiotherapy and intrathecal and systemic chemotherapy. Adequate selection of patients with greater possibilities of benefiting from more aggressive treatment is crucial. This paper, based on MEDLINE data between 1970 and 2000 , is a review of literature on this theme.

Key words: solid tumors; leptomeninges; carcinomatous meningitis.

${ }^{1}$ Médico oncologista clínico do Instituto de Previdência dos Servidores Estaduais de Minas Gerais (IPSEMG) e da ONCOMED.

${ }^{2}$ Médico oncologista clínico do Hospital Felício Rocho e Instituto de Previdência dos Servidores Estaduais de Minas Gerais (IPSEMG). Preceptor da Residência de Oncologia Clínica do Hospital Felício Rocho.

${ }^{3}$ Médica residente de Radioterapia do Hospital Belo Horizonte.

Departamentos e Instituiçōes: Hospital Felício Rocho, Serviço de Oncologia Clínica. Av. do Contorno, 9530 - Prado - Belo Horizonte - MG CEP: 30110908

Enviar correspondências para Volney Soares Lima. Rua Henrique Furtado Portugal, 145/301 - Buritis - Belo Horizonte - MG - CEP: $30455-780$

E-mail: ssala@terra.com.br 


\section{INTRODUÇÃO}

Inicialmente descrito em 1870, o acometimento metastático das leptomeninges é definido como carcinomatose meníngea ou meningite carcinomatosa ${ }^{1}$ e era considerada uma complicação rara dos tumores sólidos até recentemente. Nos últimos anos, com melhor controle sistêmico da doença neoplásica, a incidência da carcinomatose meníngea vem aumentando de maneira significativa. ${ }^{2}$

Neste artigo é feita uma revisão da literatura sobre este tema, através de pesquisa em banco de dados do MEDLINE de 1970 a 2002, baseado na necessidade crescente de uma padronização da abordagem propedêutica e terapêutica dos pacientes com suspeita clínica de meningite carcinomatosa.

\section{INCIDÊNCIA}

Aproximadamente $5 \%$ a $8 \%$ dos pacientes com câncer têm o diagnóstico de carcinomatose meníngea como complicação de sua doença. ${ }^{3}$ Potencialmente, qualquer tumor sólido pode metastatizar para as leptomeninges, ${ }^{4}$ porém o tumor mais freqüentemente relacionado a esta complicação é o câncer de mama, que nas maiores séries representa $30-60 \%$ dos pacientes com diagnóstico de carcinomatose meníngea.,

Assim como nas demais neoplasias, a incidência de carcinomatose meníngea entre os pacientes com câncer de mama está aumentando e é reportada como sendo de 2-5\%. ${ }^{1,7}$ De peculiar importância é a maior predisposição do tipo histológico lobular em se metastatizar para as leptomeninges. Esta propensão foi demonstrada em um estudo de pacientes com meningite carcinomatosa e câncer de mama, onde $58 \%$ dos pacientes apresentavam tipo histológico lobular e 33\% carcinoma ductal. ${ }^{7}$

O câncer de pulmão é o segundo tumor sólido mais freqüentemente associado a carcinomatose meníngea seguido pelo melanoma. ${ }^{8}$ No maior estudo de pacientes com diagnóstico de meningite neoplásica e câncer de pulmão não pequenas células, o tipo histológico mais freqüentemente relacionado a essa complicação foi o adenocarcinoma. ${ }^{9}$

A carcinomatose meníngea é associada à doença sistêmica em atividade em $70 \%$ a $90 \%$ dos casos, 3 sendo incomum a ocorrência de metástases para as leptomeninges como manifestação isolada da doença neoplásica.

$\mathrm{O}$ aumento da incidência de meningite carcinomatosa associada aos tumores sólidos pode ser explicado principalmente pelos avanços atingidos no tratamento sistêmico do câncer nas últimas décadas. ${ }^{10}$ Como a maior parte dos quimioterápicos utilizados tem baixa penetração pela barreira hemato-encefálica, o sistema nervoso torna-se uma espécie de "santuário" onde a doença pode persistir mesmo que controlada sistemicamente. Tem se observado também um aumento considerável da incidência de carcinomatose meníngea associada ao melhor controle das metástases cerebrais; isso é particularmente notório na ressecção de tumores localizados na fossa posterior quando o envolvimento das leptomeninges pode ser observado em cerca de $38 \%{ }^{11}$

\section{PATOLOGIA E FISIOPATOLOGIA}

A pia-máter e a aracnóide constituem as leptomeninges. $\mathrm{O}$ espaço que separa estas duas membranas é o espaço sub-aracnóideo, que contém o líquor. O neuroeixo de um indivíduo adulto contém aproximadamente $140 \mathrm{ml}$ de líquor, que é produzido pelo plexo coróide no terceiro e quarto ventrículos e nos ventrículos laterais a uma velocidade de cerca de $600 \mathrm{ml}$ por dia. ${ }^{1}$ Através dos forames de Magendie e Lusca o líquor atinge a base do cérebro e o espaço sub-aracnóideo espinhal, retornando para as cisternas basilares e deixando o neuroeixo através das granulações aracnóideas localizadas no seio sagital superior. ${ }^{3}$

Células neoplásicas podem atingir as leptomeninges através da disseminação linfática, hematogênica e pelos espaços perineurais. ${ }^{3}$ Em um estudo clássico de 28 pacientes com diagnóstico de carcinomatose meníngea verificou-se a presença de metástases para tecidos parameníngeos (principalmente vértebras) em 22 pacientes. ${ }^{12}$ Uma vez atingido o corpo vertebral as células malignas podem se disseminar através dos espaços perivasculares das veias intervertebrais e alcançar o espaço sub-aracnóideo.

A infiltração difusa das leptomeninges é a principal característica da carcinomatose meníngea, ${ }^{13}$ porém quando as células metastáticas atingem o espaço subaracnóideo elas podem invadir raízes nervosas e espaços perivasculares, dando origem a formaçôes nodulares. Pode ainda ocorrer invasão dos espaços de VirchowRobin e da pia-máter com acometimento das camadas superficiais do parênquima cerebral.8 As regiôes do sistema nervoso central mais comumente afetadas são a fossa posterior, as cisternas basilares e a superfície dorsal da cauda eqüina. ${ }^{1}$

Os achados anatomopatológicos mais freqüentemente observados em pacientes com meningite carcinomatosa incluem um espessamento fibrótico difuso das leptomeninges associado a graus variáveis de inflamação e zonas de desmielinização. ${ }^{14}$ 
De significado clínico importante é o fato de que a presença de formações nodulares no espaço subaracnóideo, decorrente do acometimento metastático das leptomeninges, é em parte responsável pela resistência à quimioterapia intratecal, pois os agentes quimioterápicos quando administrados por essa via penetram apenas alguns milímetros nos tecidos adjacentes.

\section{MANIFESTAÇ̃̃ES CLÍNICAS}

Sinais e sintomas relacionados ao envolvimento dos múltiplos níveis do neuroeixo (hemisférios cerebrais, nervos cranianos e raízes nervosas espinhais) é o quadro clínico típico da carcinomatose meníngea. ${ }^{4}$

$\mathrm{O}$ acometimento dos pares cranianos ocorre em cerca de $40 \%$ dos pacientes, sendo que os nervos responsáveis pelos movimentos oculares são os mais freqüentemente afetados. ${ }^{15}$ Os principais sintomas são diplopia, redução da acuidade auditiva e visual, paralisia facial e redução do paladar.

As metástases leptomeníngeas estão associadas a obstruçōes do fluxo liquórico em $70 \%$ dos pacientes, ${ }^{16}$ podendo ocorrer aumento da pressão intracraniana, hidrocefalia e sintomas como cefaléia, vômitos e alteraçōes do estado mental. A invasão do parênquima cerebral pelas células neoplásicas pode resultar em crises convulsivas, paresias e outros déficits neurológicos focais. ${ }^{1}$ Uma encefalopatia difusa proveniente da alteração do metabolismo cerebral ou a reduções no fluxo sanguíneo cerebral também pode estar presente nos pacientes com carcinomatose meníngea. ${ }^{10}$

Aproximadamente $48 \%$ dos pacientes apresentam sinais de acometimento das raízes espinhais como assimetria dos reflexos tendinosos, fraqueza, redução da sensibilidade, rigidez de nuca e redução do tônus retal. ${ }^{3}$ Podem ainda ocorrer dor radicular, parestesias e disfunção do reto e da bexiga. ${ }^{15}$

$\mathrm{O}$ aspecto mais importante no diagnóstico da meningite carcinomatosa é a suspeição clínica e o reconhecimento precoce do envolvimento neurológico, já que o atraso no diagnóstico e a presença de déficits neurológicos fixos são fatores de mau prognóstico.

\section{DIAGNÓSTICO}

A análise do líquor obtido por punção lombar é o principal exame complementar utilizado no diagnóstico de carcinomatose meníngea. A presença de células malignas no líquor é considerada método padrão ouro para o diagnóstico de meningite carcinomatosa, sendo os achados falso-positivos raros. ${ }^{17}$ Porém, a citologia do líquor apresenta sensibilidade insatisfatória, sendo comuns resultados falso-negativos. No maior estudo de pacientes com meningite neoplásica submetidos à autopsia, Glass et al. ${ }^{17}$ observaram que $41 \%$ dos 51 pacientes com diagnóstico de carcinomatose meníngea apresentavam citologias negativas nos exames realizados antes do óbito. $\mathrm{Na}$ série de Wasserstrom et al. ${ }^{15}$, células malignas foram identificadas no exame do líquor em $54 \%$ dos pacientes submetidos a uma punção lombar e em $80 \%$ dos pacientes submetidos a duas punçôes. A citologia do líquor é negativa em cerca de $10 \%$ dos casos, mesmo após três ou mais punçōes..$^{18}$ Deste modo, uma citologia negativa não exclui o diagnóstico de meningite carcinomatosa.

Além do exame citológico, a citometria e a análise bioquímica do líquor mostram-se alteradas na maioria dos pacientes com carcinomatose meníngea. Ocorre elevação da concentração de proteína no líquor em aproximadamente $76 \%$ dos pacientes e redução da concentração de glicose em cerca de $32 \%$ dos casos. ${ }^{4,17}$ A contagem celular encontra-se elevada em $56 \%$ dos pacientes e a maioria destas células são leucócitos mononucleares provavelmente de origem reacional. ${ }^{15}$

A análise de marcadores bioquímicos como o antígeno carcinoembrionário (CEA), desidrogenase lática (LDH) e beta-2-microglobulina no líquor vem sendo reportada em várias séries ${ }^{8,15,18}$ e pode contribuir para $\mathrm{o}$ diagnóstico de meningite carcinomatosa em alguns casos. Em um estudo de Chamberlain ${ }^{18}$ com pacientes com diagnóstico clínico e radiológico de meningite carcinomatosa e exame citológico negativo, a análise do CEA demonstrou alteração em $22 \%$ dos casos. No estudo de Wasserstrom et al. ${ }^{15}$, um nível liquórico de CEA superior a $1 \mathrm{ng} / \mathrm{ml} \mathrm{sem} \mathrm{um} \mathrm{aumento} \mathrm{concomitante}$ do nível sérico acima de $100 \mathrm{ng} / \mathrm{ml}$ foi considerado patológico. Utilizando esse critério, o CEA encontravase elevado em $21 \%$ dos pacientes, a maior parte destes com diagnóstico de câncer de mama e de pulmão.

$\mathrm{O}$ advento da ressonância nuclear magnética (RNM) trouxe importante contribuição para o diagnóstico de meningite carcinomatosa. Quando comparada com a tomografia computadorizada de crânio, a RNM demonstrou sensibilidade e especificidade superiores. ${ }^{19}$ A RNM utilizando o gadolínio como contraste detecta alteração das leptomeninges em $60-70 \%$ dos casos de carcinomatose meníngea ${ }^{3,19}$ e é o método de imagem de escolha devendo ser realizado em todos os casos de suspeita diagnóstica. As anormalidades mais freqüentemente observadas na RNM são a hipercaptação do contraste, focal ou generalizada, nos nervos cranianos, ao longo das cisternas basilares, no epêndima, na superfície do cérebro e na medula espinhal. Também pode-se observar redução no volume do parênquima 
cerebral, hidrocefalia e nódulos subaracnóideos. Apesar da superioridade da RNM quando comparada com estudo tomográfico, ambos os métodos apresentam elevada taxa de resultados falso-negativos, $30 \%$ e $58 \%$ respectivamente, ${ }^{19}$ portanto, um exame de imagem normal não exclui em definitivo o diagnóstico de carcinomatose meníngea.

Nos pacientes que necessitarem de um estudo radiológico da medula espinhal, a RNM é o exame de escolha ${ }^{8}$ devido ao seu caráter menos invasivo quando comparado à mielografia. As alterações mais comumente relatadas são os nódulos subaracnóideos, espessamento de raízes nervosas, compressão epidural e lesões intraparenquimatosas.

Um fluxo liquórico anormal é comum nos pacientes com metástases em leptomeninges. Estudos analisando o fluxo liquórico através da ventriculografia com radioisótopos mostraram que $60-76 \%$ dos pacientes com carcinomatose meníngea apresentam algum grau de obstrução ao fluxo do líquor e que a presença e a intensidade da obstrução parece estar relacionada a um pior prognóstico e à maior toxicidade do tratamento. ${ }^{16,20,21}$ A ventriculografia está indicada principalmente naqueles pacientes com exame de RNM sem evidência de lesões volumosas.

Assim, o diagnóstico de carcinomatose meníngea pode ser dado em duas situações clínicas. Na primeira, o paciente tem citologia do líquor positiva para células neoplásicas. $\mathrm{Na}$ segunda, o paciente com diagnóstico de câncer, quadro clínico compatível com acometimento neurológico multifocal e exames de imagem sugestivos de acometimento metastático de leptomeninges, mesmo com citologia liquórica negativa, que pode ser inclusive dispensada nesses casos, poderá ter o diagnóstico de carcinomatose meníngea firmado. ${ }^{8}$

\section{TRATAMENTO}

A sobrevida mediana dos pacientes com diagnóstico de carcinomatose meníngea sem tratamento é de 4 a 6 semanas, e na maioria dos casos a causa de morte é o dano neurológico progressivo. ${ }^{1} \mathrm{O}$ tratamento da meningite neoplásica tem caráter estritamente paliativo e tem como objetivo melhorar ou estabilizar os déficits neurológicos e aumentar a sobrevida. Não existe atualmente um tratamento considerado padrão, ${ }^{8} \mathrm{e}$ as principais modalidades terapêuticas empregadas são a radioterapia, a quimioterapia regional (quimioterapia intratecal) e a quimioterapia sistêmica.

Os principais fatores prognósticos nos pacientes com diagnóstico de carcinomatose meníngea são um bom performance status, doença sistêmica estável e ausência de déficits neurológicos fixos. ${ }^{22}$ Tais fatores devem ser utilizados na seleção dos pacientes com maior chance de se beneficiarem de um tratamento mais agressivo ou daqueles que são candidatos a terapia de suporte clínico.

A radioterapia tem papel fundamental na paliação dos sintomas neurológicos. A radioterapia de todo o neuroeixo está associada à elevada morbidade e a mielossupressão significativa, portanto não deve ser utilizada no tratamento das metástases leptomeníngeas. ${ }^{2}$ As áreas que apresentam lesões visualizadas através dos métodos de neuroimagem e as regióes responsáveis pelos sintomas neurológicos devem receber radioterapia focal (30 Gy em 10 a 12 frações). ${ }^{22}$ Os pacientes que apresentam obstrução ao fluxo liquórico identificada através do estudo com radioisótopos devem também receber radioterapia nos sítios de obstrução, havendo normalização do fluxo do líquor em um terço dos pacientes. $^{21}$

A quimioterapia intratecal é uma das formas de tratamento mais utilizadas nos pacientes com diagnóstico de meningite carcinomatosa, porém apresenta uma série de limitaçôes. Os poucos medicamentos que podem ser administrados por essa via com segurança, sendo eles o methotrexate, citarabina e thiotepa, possuem atividade reduzida contra os tumores sólidos que mais comumente metastatizam para as leptomeninges. Os agentes quimioterápicos administrados no espaço subaracnóideo não penetram de maneira adequada nos nódulos tumorais. ${ }^{23}$ Por fim, presença de obstrução ao fluxo liquórico aumenta a toxicidade do tratamento e pode estar associada a falhas terapêuticas. ${ }^{21}$

A quimioterapia regional pode ser realizada através de punção lombar ou através de reservatório intraventricular (reservatório de Ommaya). O clássico estudo de Shapiro et al. ${ }^{24}$ demonstrou que o methotrexate, quando administrado por via intraventricular, apresenta distribuição mais uniforme pelo espaço sub-aracnóideo. Quando o medicamento é administrado pela via lombar ele pode ser injetado inadvertidamente no espaço subdural ou epidural, ${ }^{24}$ o que diminui a concentração liquórica da droga. A punção lombar é um procedimento associado à dor e desconforto, por outro lado, a manipulação do reservatório de Ommaya está associada a complicaçôes como meningite bacteriana em 7,5\% dos casos ${ }^{25}$ e à obstrução do cateter em 5-9\%. ${ }^{3,25}$ Apesar das vantagens farmacocinéticas favorecendo a via intraventricular quando comparado com a punção lombar, não existem estudos randomizados em pacientes adultos que demonstrem uma melhor sobrevida com a utilização dessa via. ${ }^{8}$

Entre os medicamentos disponíveis para o uso intraventricular o methotrexate é o mais utilizado. A 
concentração do methotrexate de $1 \times 10-6 \mathrm{~mol} / \mathrm{L}$ é considerada como terapêutica na maioria dos estudos, ${ }^{26}$ e pode persistir por até 48 horas após administração da droga. ${ }^{24} \mathrm{O}$ methotrexate que não é metabolizado no sistema nervoso central, atinge a circulação sistêmica através do plexo coróide, podendo provocar neutropenia e mucosite em alguns pacientes. O ácido folínico administrado por via oral pode reduzir a toxicidade sistêmica do methotrexate sem comprometer a sua eficácia. ${ }^{22} \mathrm{O}$ methotrexate geralmente é administrado na dose de $12 \mathrm{mg}$ duas vezes por semana no total de 8 doses seguido de uma dose semanal por quatro semanas e posteriormente uma dose de manutenção mensal. ${ }^{1}$ Entretanto, não existem estudos randomizados comparando a terapia em bolus semanal com o esquema de tratamento utilizando a relação concentração/tempo descrita por Chamberlain. ${ }^{9}$ Em um estudo retrospectivo, Fizazi et al. ${ }^{27}$ demonstraram que o aumento da dose de methotrexate pode se traduzir em maior taxa de resposta, contudo, a literatura carece de estudos randomizados comparando as várias doses utilizadas. Hitchins et al. ${ }^{28}$, em um estudo prospectivo e randomizado com 44 pacientes, comparou a terapia intratecal utilizando o methotrexate como droga isolada com a combinação de citarabina mais methotrexate. A taxa de resposta global foi de $55 \%$ e a sobrevida mediana de 8 semanas, não havendo diferença significativa entre os dois braços.

O thiopeta é um agente alquilante rapidamente metabolizado após a administração intratecal. ${ }^{3}$ No tratamento da carcinomatose meníngea, o thiotepa é administrado na dose de $10 \mathrm{mg} 3$ e geralmente é utilizado nos pacientes que não toleram o methotrexate ou que não respondem ao tratamento inicial. Estudo randomizado e prospectivo comparando a administração intraventricular do methotrexate e thiotepa em pacientes com diagnóstico de meningite neoplásica sem história de tratamento prévio não observou diferença na eficácia entre os dois regimes. ${ }^{29} \mathrm{~A}$ sobrevida mediana nos pacientes que receberam methotrexate foi de 15,9 semanas e naqueles tratados com thiopeta foi de 14,1 semanas. A toxicidade foi semelhante nos dois grupos, porém os pacientes que fizeram uso do methotrexate apresentaram maior incidência de neurotoxicidade ( $\mathrm{p}: 0.008$ ).

A citarabina é adequada para o uso intratecal visto que a sua principal via de metabolismo, a conversão em uracil-arabinosídeo pela citidinadeaminase, não ocorre no espaço sub-aracnóideo. ${ }^{3}$ Entretanto a citarabina não possui atividade contra a maioria dos tumores sólidos, sendo utilizada principalmente nas leucemias e nas infiltraçôes meníngeas pelos linfomas. ${ }^{1}$ A citarabina de liberação sustentada foi recentemente aprovada para uso em pacientes com meningite linfomatosa ${ }^{22,30}$ e possui como principal vantagem manter níveis terapêuticos da droga no líquor por mais de 14 dias após uma dose única de $50 \mathrm{mg}$. Glantz et al..$^{31}$ reportaram os resultados de um estudo randomizado comparando o uso do methotrexate e a citarabina de liberação sustentada, ambas as drogas administradas por via intratecal em pacientes com diagnóstico de meningite neoplásica por tumores sólidos. Neste estudo, a mediana do tempo de progressão neurológica foi significativamente maior no braço tratado com citarabina de liberação sustentada (p: 0.007), porém não houve diferença estatisticamente significativa em relação à sobrevida mediana.

A injeção de drogas no espaço sub-aracnóideo está associada a uma série de complicações. A ocorrência de meningite asséptica é reportada em $10 \%$ a $43 \%$ dos pacientes $^{15,25}$ e é caracterizada pelo início de febre, cefaléia, confusão mental, náuseas, vômitos e meningismo algumas horas após a administração do quimioterápico. $\mathrm{O}$ exame do líquor nesses casos revela pleocitose com culturas negativas e ocorre regressão dos sintomas após uso de corticoterapia oral. ${ }^{25}$ Mesmo com o uso do ácido folínico, cerca de $8 \%$ dos pacientes desenvolvem toxicidade sistêmica relacionadas ao methotrexate como mucosite e mielossupressão. ${ }^{15} \mathrm{~A}$ leucoencefalopatia, principal complicação tardia do tratamento intratecal, ocorre com maior freqüência naqueles que receberam methotrexate e radioterapia cerebral e é observada em cerca de $10 \%$ dos pacientes. ${ }^{32}$ O quadro clínico é caracterizado por apatia, perda das funçōes cognitivas, hemiparesias e o estudo tomográfico evidencia hipodensidades periventriculares. ${ }^{32}$

$\mathrm{O}$ uso de quimioterápicos sistêmicos na carcinomatose meníngea era tido como ineficaz, porém a quimioterapia sistêmica apresenta uma série de vantagens e vem sendo cada vez mais utilizada. Os medicamentos administrados por via sistêmica conseguem penetrar bem nos volumosos nódulos subaracnóideos, que têm seu crescimento sustentado pela neovascularização. ${ }^{18}$ Elimina-se também o desconforto causado por punçôes lombares múltiplas e os riscos associados à manipulação dos reservatórios intraventriculares. Além disso, estudos retrospectivos não demonstraram vantagem do uso da quimioterapia intratecal quando comparada com radioterapia isolada6 ou com o tratamento sistêmico. ${ }^{7,32}$

O methotrexate quando administrado em doses elevadas por via sistêmica atinge concentrações liquóricas terapêuticas semelhantes às atingidas com o tratamento intratecal. ${ }^{26}$ No estudo de Glants et al..$^{26}$ o methotrexate foi administrado a cada 14 dias na dose $\mathrm{de} 8 \mathrm{~g} / \mathrm{m}^{2}$ seguido de resgate com ácido folínico. A taxa de resposta citológica completa foi de $81 \%$ e a sobrevida mediana 
foi de 13,8 meses enquanto que no grupo controle tratado com methotrexate intratecal a sobrevida mediana foi de 2,3 meses (p: 0,003). Em um outro estudo, Postbus et al..$^{33}$ utilizou etoposide em altas doses $\left(1 \mathrm{~g} / \mathrm{m}^{2}\right.$ por 3 dias a cada 4 semanas) em cinco pacientes com diagnóstico de carcinoma pulmonar de pequenas células e meningite neoplásica, observando uma melhora neurológica completa em três pacientes. Recentemente, Boogerd ${ }^{34}$ relatou o tratamento de pacientes com metástases leptomeníngeas de câncer de mama utilizando terapia hormonal e radioterapia com bons resultados.

Outros agentes em investigação no tratamento da carcinomatose meníngea incluem a nitrosuréia ACNU, ${ }^{35}$ o agente alquilante diaziquona, ${ }^{36}$ a temozolomida ${ }^{37}$ e o teniposide. ${ }^{38} \mathrm{O}$ trastuzumab, anticorpo monoclonal utilizado em pacientes com câncer de mama metastático, atinge concentrações liquóricas 300 vezes menor que as concentrações plasmáticas, e até o presente momento, não demonstrou ser efetivo no tratamento das metástases leptomeníngeas. ${ }^{39}$

\section{CONSIDERAÇÕES FINAIS}

O diagnóstico de carcinomatose meníngea implica em prognóstico reservado para a grande maioria dos pacientes com tumores sólidos. A sobrevida mediana é de 2 a 4 meses 7,8 e em cerca de $50 \%$ dos pacientes tratados a principal causa de morte é a progressão da doença sistêmica. ${ }^{15}$ Após o diagnóstico de meningite neoplásica, grande parte dos pacientes não irá se beneficiar de um tratamento agressivo. Naqueles pacientes com performance status comprometido e nos que apresentam doença sistêmica em progressão devese considerar o uso de radioterapia paliativa e de analgésicos.

Em relação ao melhor tratamento para os pacientes com meningite neoplásica, devemos considerar alguns pontos na literatura referente a esse tema. Existem poucos estudos prospectivos randomizados com poder suficiente para comparar as várias opções terapêuticas. A maioria dos estudos incluem pacientes com carcinomatose meníngea de tipos histológicos variados o que dificulta a determinação de fatores prognósticos e a comparação de resultados. Ainda não estão definidos qual o melhor esquema de tratamento intratecal, a melhor via para esse tratamento e nem a sua duração. Por fim, a quimioterapia sistêmica é promissora e ainda não foi adequadamente comparada às outras formas de tratamento.

Com o aumento crescente na incidência da carcinomatose meníngea como complicação tardia dos tumores sólidos, faz-se necessário enfatizar a importância de seu diagnóstico precoce e estimular a realização de estudos clínicos bem conduzidos para definição do melhor tratamento.

\section{REFERÊNCIAS BIBLIOGRÁFICAS}

1. Grossman S. Advances in the treatment of central nervous system metastases: treatment of neoplastic meningitis. 2001 Educational Book of the 37th Annual Meeting of the American Society of Clinical Oncology; 2001 May 12-15; San Francisco, CA. p. 598-604.

2. DeAngelis LM. Diagnosis and treatment of leptomeningeal metastasis. 2002 Educational Book of the 38th Annual Meeting of the American Society of Clinical Oncology; 2002 May 18-21; Orlando, FL. p. 369-74.

3. Jayson GC, Howell A. Carcinomatous meningitis in solid tumors. Ann Oncol 1996;7(8):773-86.

4. Van Oostenbrugge RJ, Twijnstra A. Presenting features and value of diagnostic procedures in leptomeningeal metastases. Neurology 1999;53(2):382-5.

5. Pfeffer MR, Wygoda M, Siegal T. Leptomeningeal metastases - treatment results in 98 consecutive patients. Israel J Med Sci 1988;24:611-8.

6. Bokstein F, Lossos A, Siegal T. Leptomeningeal metastases from solid tumors. Cancer 1998;82(9):1756-63.

7. Jayson GC, Howell A, Harris M, Morgenstern G, Chang J, Ryder D. Carcinomatous meningitis in patients with breast cancer: an aggressive disease variant. Cancer 1994;74(12):3135-41.

8. Chamberlain MC. Neoplastic meningitis: a guide to diagnosis and treatment. Cur Opin Neurol 2000;13:641-8.

9. Chamberlain MC, Kormanik RN. Carcinoma meningitis secondary to non-small cell lung cancer. Arch Neurol 1998;55:506-12.

10. Sagar SM. Carcinomatous meningitis: it does not have to be a death sentence. Oncology 2002;16(2):237.

11. DeAngelis LM. The Sagar article rewiewed. Oncology 2002;16(2):237.

12. Kokkoris CP. Leptomeningeal carcinoimatosis. How does cancer reach the pia-arachnoid? Cancer 1983;51:154-60.

13. Gonzalez-Vitale JC, Garcia-Bunuel R. Meningeal carcinomatosis. Cancer 1976;37:2906-11.

14. Olson ME, Chernik NL, Posner JB. Infiltration of the leptomeninges by systemic cancer. Arch Neurol 1974;30:122-37.

15. Wasserstrom WR, Glass P, Posner JB. Diagnosis and treatment of leptomeningeal metastases from solid tumors: experience with 90 patients. Cancer 1982;49:759-72.

16. Mason WP, Yeh SDJ, DeAngelis LM. Indiumdiethylenetriamine pentaacetic acid cerebrospinal fluid flow studies predict distribution of intrathecally administered chemotherapy and outcome in patients with leptomeningeal metastases. Neurology 1998;50(2):438-44. 
17. Glass JP, Melamed M, Chernik NL, Posner JB. Malignant cells in cerebrospinal fluid (CSF): the meaning of a positive CSF cytology. Neurology1979;29:1369-75.

18. Chamberlain MC. Cytologically negative carcinomatous meningitis: usefulness of CSF biochemical markers. Neurology 1998;50(4):1173-5.

19. Chamberlain MC, Sandy AD, Press GA. Leptomeningeal metastasis: a comparison of gadolinium-enhanced $M R$ and contrast-enhanced CT of the brain. Neurology 1990;40:435-8.

20. Glantz MJ, Hall WA, Cole BF, Chozick BS, Shannon CM, Wahlberg L, et al. Diagnosis, management and survival of patients with leptomeningeal cancer based on cerebrospinal fluid-flow status. Cancer 1995;75(12):2919-31.

21. Chamberlain MC, Corey-Bloom JC. Leptomeningeal metastases: Indium-DTPA CSF flow studies. Neurology 1991;41:1765-9.

22. Glantz M. Carcinomatous and lymphomatous meningitis. In: UpToDate 10.2. 2002.

23. Gilbert MR. The Sagar article reviewed. Oncology 2002;16(2):237.

24. Shapiro WR, Young DF, Mehta BM. Methotrexate: distribution in cerebrospinal fluid after intravenous, ventricular and lumbar injections. N Engl J Med 1975;293(4):161-6.

25. Chamberlain MC, Kormanik PA, Barba D. Complications associated with intraventricular chemotherapy in patients with leptomeningeal metastases. J Neurosurg 1997;87:694-9.

26. Glantz MJ, Cole BF, Recht L, Akerley W, Mills P, Saris S, et al. High-dose intravenous Methotrexate for patients with nonleukemic leptomeningeal cancer: is intrathecal chemotherapy necessary? J Clin Oncol 1998;16(4):1561-7.

27. Fizazi K, Asselain B, Vincent-Salomon A, Jouve M, Dieras $\mathrm{V}$, Palangie T, et al. Meningeal carcinomatosis in patients with breast carcinoma. Clinical features, prognostic factors, and results of high-dose intrathecal methotrexate regimen. Cancer 1996;77(7):1315-23.

28. Hitchins RN, Bell DR, Woods RL, Levi JA. A prospective randomized trial of single-agent versus combination chemotherapy in meningeal carcinomatosis. J Clin Oncol 1987;5:1655-62.

29. Grossman SA, Finkelstein DM, Ruckdeschel JC, Trump DL, Moynihan T, Ettinger DS. Randomized prospective comparison of intraventricular methotrexate and thiopeta in patients with previously untreated neoplastic meningitis. J Clin Oncol 1993;11:561-9.

30. Glantz MJ, LaFollette S, Jaeckle KA, Shapiro W, Swinnen L, Rozental JR, et al. Randomized trial of a slow-release versus a standard formulation of cytarabine for the intrathecal treatment of lymphomatous meningitis. J Clin Oncol 1999;17(10):3110-6.

31. Grantz MJ, Jaeckle KA, Chamberlain MC, Phuphanich S, Recht L, Swinnen LJ, et al. A randomized controlled trial comparing intrathecal sustained-release Cytarabine (DepoCyt) to intrathecal Methotrexate in patients with neoplastic meningitis from solid tumors. Clin Cancer Res 1999;5:3394.

32. Boogerd W, Hart AAM, van der Sande JJ, Engelsman E. Meningeal carcinomatosis in breast cancer. Prognostic factors and influence of treatment. Cancer 1991;67:1685-95.

33. Postmus PE, Haaxma-Reiche H, Berendsen HH, Sleijfer DT. High dose etoposide for meningeal carcinomatosis in patients with small cell lung cancer. Eur J Cancer Clin Oncol 1989;25:377-8.

34. Boogerd W, Dorresteijn LDA, van der Sande MD, Gast GC, Bruning PF. Response of leptomeningeal metastases from breast cancer to hormonal therapy. Neurology 2000;55(1):117-9.

35. Levin VA, Chamberlain M, Silver P, Rodriguez L, Prados M. Phase I/II study of intraventricular and intrathecal ACNU for leptomeningeal neoplasia. Cancer Chemother Pharmacol 1989;23(5):301-7.

36. Berg SL, Balis FM, Zimm S, Murphy RF, Holcenberg J, Sato J, et al. Phase I/II trial and pharmacokinetics of intrathecal diaziquone in refractory meningeal malignancies. J Clin Oncol 1992;10:143-8.

37. Friedman HS. Temozolomide in early stages of newly diagnosed malignant glioma and neoplastic meningitis. Semin Oncol 2000;27(3):35-40.

38. van der Graaf WT, Haaxma-Reiche H, Burghouts JT, Postmus PE. Teniposide for meningeal carcinomatosis of small cell lung cancer. Lung Cancer 1993;10:247-249.

39. Baculi RH, Suki S, Nisbett J, Leeds N, Groves M. Meningeal carcinomatosis from breast carcinoma responsive to Trastuzumab. J Clin Oncol 2001;19(13):3297-8. 